\title{
The far-seeing planning systems and models for the construction management
}

\author{
Lubov Petrenko ${ }^{1}$, Svetlana Manzhilevskaya ${ }^{1}$, Aleksandr Shilov ${ }^{1}$ and Al-Hajj Ali Abdullah \\ Saleh ${ }^{1, *}$ \\ ${ }^{1}$ Don State Technical University, Academy of Civil Engineering and Architecture, 344010, Rostov- \\ on-Don, Gagarina square, 1, Russia
}

\begin{abstract}
The scientific basis of the organization of construction production involves the development of innovative models and methods of construction planning. The article studies the issues of perspective planning. After several years of research, the authors propose a new approach to forecasting and long-term planning. The authors consider the construction as a holistic design and realistic complex. As defined by the Bank of World, a project is a set of interrelated activities designed to achieve objectives within a given time frame and budget, with clearly defined objectives. In the article the mathematical model allowing to plan construction production for long term and to operate it is offered. The proposed method makes it possible to effectively and ergonomically solve the problems of construction plan and construction management.
\end{abstract}

\section{Introduction}

In the course of planning improvement the far-seeing plan goes into the main management tool of the economy. In this regard it is necessary to give scientific credence for the far-seeing plan and to determine the research methods of calculations for the effective far-seeing plan development at all levels. In order to have the far-seeing plan accorded with allotted task it is sensible to develop the far-seeing planning systems and models.

This article describes the far-seeing planning systems and models development for the construction management. These models help to prove the choice of production processes for the construction and to inculcate the innovated objects. The first choice calculation can be done only with the help of the operations research. The operations research methods adaptation and the operating personnel activity stimulation help to find and study the reserves, which can have influence on the flow processes optimization in future.

Investment management problem in the construction industry is very relevant today. It examined many scientists.

In the O.A Pobegaylov and O.V Klyuchnikova [1] referred to the close relationship with the investment policy of the organization and management of construction, the use of traffic management techniques, including linearly extended objects of underground construction .

\footnotetext{
* Corresponding author: oc41@bk.ru
} 
LK Petrenko, S.E Manzhilevskaya and D.A Bogomazyuk [2] consider the problem of innovation in construction technologies and investments in the sector, taking into account the implemented innovations of this type. O.A.Pobegaylov, O.E.Gaybaryan, G.I.Myasischev [3] and talk about the role of document and information management companies when choosing an investor-customer organization - contractor.

\section{Materials and Methods}

The objective function is considered there as the group of the economic factors. The most important factors are:

- maximum profit;

- maximum bottom-line profit;

- maximum net currency income;

- maximum income;

- maximum common costs;

- maximum labor costs;

- $\quad$ maximum certain production output (according to the national economy need);

- minimum payroll;

- minimum investment.

The first three factors are summarized ones (for construction materials industry), the others are particular factors. It is clear that only one factor is used as the objective function for optimization model calculation, then it is necessary to take into account the other factors requirements as the system of extra conditions. IAs the national economy efficiency is determined as the maximum national income increment, so the each certain sub system must purpose the maximum profit or bottom-line profit receiving. The essential factor of making correct decision is the usage of the effective prices and estimates according to the task order[4,5].

As extra conditions for model calculation we can consider the upper limit values of such economic indicators as the volume of raw materials, production capacities, capital investments, etc. Along with them, a certain role is played by intermediate deliveries, product quality, market requirements, problems associated with the use of production facilities and premises, etc. In addition, the amount of capital investment, the wage fund, etc. may be limited. The latter is valid provided that these indicators have not previously been selected as an objective function.

\section{Results}

The Pichler matrix model is used as the base for calculation the. It is considered as the cost and output matrix of certain manufacturing complex:

$$
K=\left(\begin{array}{ll}
K 11 & K 12 \\
K 21 & K 22
\end{array}\right)
$$

A model based on the actual performance equipment is used here, since most of the indexes in it have a value of 0 . It is advantageous in terms of the reduction of time consumption for optimization [6-8]. Theoretically, you can use mathematical models based on the output of products or the costs incurred. For a better understanding of the essence of the issue under consideration, let us first assume that all technological processes have already been selected. The cost and output matrix provides the following relationship: 


$$
\left(\begin{array}{l}
f \\
Q
\end{array}\right)=\left(\begin{array}{ll}
K 11 & K 12 \\
K 21 & K 22
\end{array}\right)\left(\begin{array}{l}
d \\
t
\end{array}\right)
$$

where $f$ - commercial output vector (This includes also products received on intermediate deliveries; In this case the corresponding components have a value of 0 );

a - input vector;

d - physical output vector;

t - influencing quantities or arguments vector. One of the most important influencing factors for the next calculation is time, because the certain cost values, for example expenditures, changes prorate duration of considered correspondence period.

During optimal planning - not yet far-seeing planning it is available to define the linear optimization problem with the help of the cost and output matrix[3,5-7].

We simplify the computations and choose the profit indicator as the objective function. We set the parameters in the cost and output matrix in such a way that the output indicators are positive and consumption indicators are negative.

This expression has the form:

$$
P^{*}=\left(P^{*} f_{0} P Q\right)
$$

This expression we put into the matrix. Thus the final product prices and different types of expenditures vector has the form:

$$
\left(P f_{0} P^{\prime} Q\right)\left(\begin{array}{ll}
K 11 & K 12 \\
K 21 & K 22
\end{array}\right)
$$

If we reduce the sundry expenses (salaries \& wages, repair costs, auxiliary supplies costs , depreciation charges) from this line, divided into the capacity and influence parameters, and multiply, them like this:

$$
g^{2}=\left(g^{2} d^{2} t\right)\left(\frac{d}{t}\right)
$$

so we will get the quantitative assessment of the profit, which includes the committed cost. It is obvious that the committed costs don't influence on the optimality index, so as it stands we shouldn't take them into account. It is difficult to divide sundry expenses into the capacity and influence parameters, so the dividing process will be lacking detail. Most of these expenses are in direct ratio to the time consumption. We will be right about if we suppose that these values are proportional to each other; at least it is true for the depreciation charges [9].

So the optimum problem will have the form:

$$
\begin{aligned}
& Z=g\left(\frac{d}{t}\right) \rightarrow \max \| \\
& d \leq d^{0}{ }_{0} K_{21} d+K_{22} t \leq a^{0},
\end{aligned}
$$

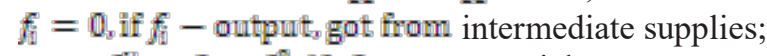

$$
\begin{aligned}
& f_{i}^{L} \leq f_{i} \leq f_{i}^{0} \text { if } f_{i}-\text { commercial output; } \\
& d \geq 0 \text {. }
\end{aligned}
$$

where $d^{\mathbb{Q}}$ - maximum sustainable yield vector; $a^{\mathbb{D}}$ - maximum different expenses amount vector; $f-f$ vector element, $f_{i}^{L}$ and $f_{i}^{\square}$ - desired high and low limit of commercial output production volume. $F_{0}$ - values can be linearized by $\underline{d \text { - }}$ and $\underline{t}$ - elements using. $\underline{T \text { - elements }}$ should be determined and evaluated before optimization performing, as such parameters, like time, temperature can't be optimized.

The application of this calculation for the far-seeing planning is possible only if the technological processes have already been selected. If we consider the fact that during a relatively long period of the far-seeing plan action, technological processes can still be 
under developing, and we don't know about them now, the central task will consist of choosing the most optimal technologies.

In this regard, it is necessary to include in the model all possible variants of the phases of technological processes (obtaining identical results from the use of different technologies at a certain phase of the process), and also to take into account all possible links with other production units of the industry. As for other extra conditions, the indexes, which take into account capital investments, a payroll, etc in the calculation, can be considered as values, which depend only on technology and should be recorded in the corresponding column of time parameters. In reality, these indexes also depend on the size of the industrial plant, and thus on the performance [10-13]. However, it will be shown below that such a relationship can be taken into account by entering several columns of time parameters into calculation. At first we should consider the simplest case where the actual performance of each of the technological processes at our disposal should vary in close limits, and the amount of costs and auxiliary means necessary for the introduction of the technology is also known and accurately determined. In such a case, only one column of time parameters is prepared for each technological process. Of course, these costs should not be taken into account if the corresponding technology is rejected and therefore not realized. It should also exclude from the matrix other possible columns of parameters [1,14,15].

As can be seen from the above, it is appropriate to add the objective into the optimization problem, which must be taken into account in each column of parameters:

$$
t_{1}=\left\{\begin{array}{c}
0, \text { if performance of the process is } 0_{3} \\
\text { actuating value is given in advance, if performance of the process is above } 0 .
\end{array}\right.
$$

where $t_{1}$ - datum value of the column of parameters, in other words vector $\underline{t}$ elements. If we multiply the parameters value of the column and the value of a is given in advance, so the objective stated above, will have a simpler form:

$$
t_{1}=\left\{\begin{array}{c}
0, \text { if performance of the process is } 0 ; \\
1_{s} \text { if performance of the process is above } 0 .
\end{array}\right.
$$

It should be noted that the certain minimal load of device must take place, as otherwise it operates cost-ineffective. If we suppose that $t_{1}$-objective is according to $d_{\Omega}$ physical output and a technological process is performed only under $d_{\mathrm{d}}^{\mathrm{W}} \leq \mathrm{d}_{\mathrm{L}} \leq d_{\mathrm{d}} \mathrm{Q}$ condition ( $d_{\mathbb{W}}^{\mathbb{Q}}$-production capacity), so the extra objective will have this form:

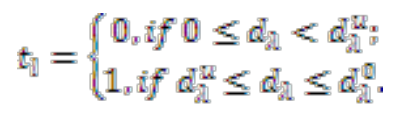

$d_{2 \mathrm{~W}}^{\mathrm{W}}$ and $d_{\mathrm{S}}^{\mathbb{Q}}$ values are limits, which determine the range of the physical output index. However, the linear optimization methods of solution can neglect such objectives, so a need arises in corresponding transformations. Next we will consider the analysis of necessary transformations and also the set of conditions which can assist in problem solving with the help of certain methods.

The objective (9) has following conditions:

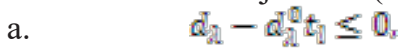
b. $\quad d_{\mathrm{d}}-d_{\mathrm{W}}^{\mathbb{W}} t_{\mathrm{D}} \geq 0$
c. $\quad 0 \leq t_{n} \leq 1_{x}$
d. $\quad t_{1}$-integer number.

It stems from the fact that:

1. Where $0 \leq d_{\mathrm{M}}<d_{\mathrm{N}}^{\mathrm{W}}$ and $t_{1}=1$, it's nonsatisfiability of (b) condition.

In this situation it's followed from (a) condition, that $d_{\mathrm{h}}=0$;

2. Where $d_{d \mathbb{L}}^{\mathbb{W}} \leq d_{\mathrm{N}} \leq d_{\alpha}^{\mathbb{Q}}$ and $t_{1}=0$, it's nonsatisfiability of (a) condition; 
According to (c) and (d) conditions the task in hand belongs to mixed-integer type: $d_{2}$ performance indexes are persistent, but $t_{1}$-conditions are discrete variables. Here we also have the $0-1$ type of the optimization, as the discrete variables can take on value either 0 or 1.

If we have r-different capacity measures, we introduce $r$ of $t_{\text {lij }}$ - discrete variables, where $i=1$ (9)r into the $d_{\mathbb{Q}}$-physical output equation. If we denote high and low limits of certain device physical output indexes by $d_{\delta}^{\mathbb{Q}}$ and $d_{\mathcal{L}}^{\mathrm{L}}$, where $i=1(9) \mathrm{r}$, so we can get following extra conditions for certain technology:
1. $d_{\mathrm{d}}-\sum_{\mathrm{i}=1} r \mathrm{~d}_{\mathrm{i}}^{\mathbb{0}} \mathrm{t}_{\mathrm{ii}} \leq 0_{x}$
2. $\quad d_{\mathrm{N}}-\sum_{\mathrm{i}=1} r d_{\mathrm{W}}^{\mathbb{W}} t_{\mathrm{li}} \geq 0$,
3. $0 \leq t_{\mathrm{li}} \leq 1$ where $i=1(9) \mathrm{r}$,
4. $t_{\text {ii }}$ - integer number where $i=1(9) \mathrm{r}$.

These conditions are completely corresponded to the conditions, described above (a-d). Thus, in this case we also have mixed-integer method of linear optimum problem of $0-1$ type solving[4,16,17].

We will have the other site of task construction, if we consider the operating of several equal devices during the technological process introduction. Suppose that we can invest in $s$ or less of single-type devices, necessary for the technological process assimilation. We determine $d_{\alpha}^{\underline{\mu}}$ and $d_{\alpha \mathbb{Q}}^{\mathbb{Q}}$ as minimum and maximum capacity of one of these devices. Here we have the special case of problem solving, where you can have just only one discrete variable value $-t_{1}$. It follows the extra conditions:
a. $\quad d_{2}-d_{2}^{\mathbb{Q}} t_{1} \leq 0_{x}$
b. $\quad d_{2}-d_{2}^{K} t_{1} \geq 0$
c. $\quad 0 \leq t_{1} \leq s_{x}$
d. $t_{n}$-integer number.

In this way, we have the standard linear optimum problem, which can be solved by the mixed-integer method as the discrete variables can have value different from 0 or 1 . It should be noted that in practice problem solving may be mixed. It is necessary to consider that there are two main groups of problem solving methods, such as the truncation methods and the branch and bound methods. When we consider the branch and bound methods we should take into account the set of extra conditions[18].

These conditions help to single out either one of the possible technologies or exceptionally correlated technologies and connect them.

In the first case, as a rule, these conditions (where $t_{\mathbb{1} 1} \mathrm{t}_{\mathbb{1 k}}$ - are the 0-1 type discrete variables of singled out technology at the one stage of the flow process) have this form:

$$
t_{11}+t_{12}+\cdots+t_{i k} \leq s
$$

In this connection we suppose that it's taken into account maximum $s$-technologies from $k$-possible ones. Generally, $\mathrm{s}=1$.

In the second case these conditions have this form:

$$
t_{j 1}+t_{j 2}+\cdots+t_{j=2}=n_{s} \text { if this union } \neq 0
$$

In this connection $t_{j 1} \ldots t_{j n s}$ are the 0-1 type discrete variables of correlated technologies at the different stages of the flow process. The correct process model development provides for the considering optimum connections.

1. Conditions of constraint. These are conditions between two stages of the flow process. If $t_{\mathbb{1 1}} \ldots t_{\mathbb{R}}$-values and $t_{j 1} \ldots t_{j \mathrm{j}}$-values are all discrete variables of technologies at two correlated stages of the flow process, so these conditions have this form: 


$$
\begin{aligned}
& t_{\mathbb{1}}+\cdots+t_{\mathbb{R}}=0 \leftrightarrow t_{j 1}+\cdots+t_{j 1}=0, \\
& t_{11}+\cdots+t_{l k}=0 \leftrightarrow t_{j 1}+\cdots+t_{j 1} \neq 0 .
\end{aligned}
$$

2. Conditions of minimization. Usually the lower limit values of the far-seeing planning optimization problem are defined as:

It follows the extra condition:

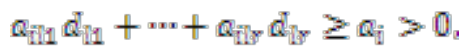

$$
t_{\prod 1}+\cdots+t_{\prod y} \geq 1
$$

So far as there is a superior limit for $d_{\mathbb{1} \text {, }}$, it may become necessary to have two or more positive values of the physical output indexes. For this reason condition of minimization has this form:

$$
t_{11}+\cdots+t_{\prod y} \geq u_{x}
$$

In this connection, it is not necessary that the columns of parameters, recorded in the left part, relates to the technologies of the one stage of the flow process.

3. Conditions of maximality. The upper limit values can be defined by the following equation (inequality):

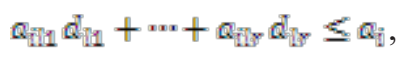

It is obvious that we have the similar condition as (13), but with opposite in sign. Another real opportunity of upper delimitation is the construction costs and capital costs recording in $t$-columns of calculation, hence we have the extra conditions of this form:

$$
b_{\mathbb{T I 1}} t_{\mathbb{i 1}}+b_{\mathbb{i l y}} t_{i y} \leq b_{i x}
$$

In this situation if we consider the indexes of left and right parts, we can get delimitation as:

$$
t_{\text {li1 }}+x+t_{\text {lip }} \leq w(w<v),
$$

Otherwise we can perform checks, using the equation (16).

\section{Discussion}

Ralph Gomory was the first who developed a method of solving linear optimization problems of a discrete type suitable for practical use. The solution of the problem with the help of the proposed Gomory's method is carried out firstly without taking into account the requirements of integrality. After this new extra conditions are consistently introduced, they make the computed solution unacceptable. However, all those moments that can be considered as a possible solution that takes into account the requirements of integrality, can be adopted. Then the model is again optimized. The whole process continues until an integer solution is found. However, all these actions don $\mathrm{t}$ make the analyzed method completely scrupulous. The most significant limitations of this method are as follows.

First of all, there is a gradual increase in the volume of the problem in the process of solving with each successor step. Although such an increasing in volume stays within (otherwise the extra conditions are out of court), it causes inconvenience with the programming technique usage[19].

Then it is difficult to define the desired number of step of calculation.

Finally, the serious complications are caused by round off error. During computing recalculations the integers (because of round off error) stop be recognized. The rounds off errors are unanalyzable and there is no practical method of their quantitative estimation. In 
this regard it is difficult to define the integer. In case of an unsuccessful choice computer can't find the problem solution, although it is possible, in theory.

Many properties of linear programming can be interpreted as polyhedron properties, thus, geometrically formulated and proved. This solution is the basis of several methods for solving integer programming problems[4,20].

Increasingly the branch and bound method is used for solving common combinatorial optimization problems, which include integer linear optimization methods. The goal of a branch and bound method is to find a value $x$ that maximizes or minimizes the value of a real-valued function $f(x)$, called an objective function, among some set $S$ of admissible, or candidate solutions. The set $S$ is called the search space, or feasible region. The rest of this section assumes that minimization of $f(x)$ is desired; this assumption comes without loss of generality, since one can find the maximum value of $f(x)$ by finding the minimum of $g(x)=$ $-f(x)$.

\section{Conclusions}

Modern investment management cannot function effectively in the absence of relevant information and communication support. The most rational method of management of the investment strategy is cognitive control method. In order to effectively invest in the construction requires the use of innovation. At the decision of specific targets the simulation system must ensure full compliance with the input and output data of these tasks to the requirements of the estimated parameters. It is necessary that the output of the programs is a model designed according to current standards in the form of settlement, graphic, formalized text materials. The documentation formed in this way without any improvements could be used by the contractor in the construction of the facility.

\section{References}

1. O.V. Kliuchnikova, O.A. Pobegaylov, Procedia Engineering. 150, pp. $2168-2172$ (2016).

2. L.K. Petrenko, S.E. Manzhilevskaya, D.O.Procedia Engineering. 150, 2302 - 2307 (2016)

3. O.A. Pobegaylova, G.I. Myasishcheva, O.E. Procedia Engineering. 150, pp.2173-2177 (2016)

4. G.Z Gabidullina. PhD Thesis, Ufa (2013).

5. L.E.Basovskiı̌, Basovskaya E.N. Economic evaluation of investments, INFRA-M, Moscow (2008).

6. T.S. Kolmykova Investment analysis, INFRA-M, Moscow (2009).

7. A.I. Fazrahmanova PhD Thesis, Ufa (2013).

8. M. W. Schaefer Return of Influence: The Revolutionary Power of Klout, Social Scoring, and Influence Marketing (McGraw-Hill, 2001)

9. P. Lazarsfeld The People's Choice. (McFadden Publications, 1998)

10. M. Speyer Identifying IT Buyers' Hidden Influencers: Finding and Nurturing Your Brand Presence Beyond Your Formal Channels Forrester Research, (1962)

11. P. Masse Optimal investment decisions. Englewood Cliffs N.J., (1962)

12. E. B. Nikolenko Organizational-economic mechanism of investment support. Ufa, 174 (1987) 
13. P. R. Keeling Horizontal and Vertical structures: The dynamics of organization in highereducation Liberal Education 93, 4, 22-31(2007)

14. E.Shirina, E.Gaybarian, G. Myasischev Effective management of construction company in terms of linguistic communication IOP Conf. Series: Earth and Environmental Science 90, 012077 (2017)

15. V. V. Kostiuchenko The System Organization and Management of Construction, RGSU, 210 (2014)

16. O. A. Pobegailov Investments in Unstable Economical System. Terra Economicus scholar, 2-2, 10, 35-38 (2012)

17. A.V. Babanov Classification of factors shaping the investment attractiveness of the region, "Economic Journal", Russian State Humanitarian University. 4(28), 107-114 (2012)

18. I.A. Blank Fundamentals of Financial Management, Nika-Tsentr (1999)

19. A.F.Wall, R.P. Keeling Horizontal and Vertical structures: The dynamics of organization in higher education, Liberal Education, 4(93), 22-31(2007)

20. I.L. Leontiev Modeling innovation and investment development of socially significant objects on the territory: Abstract. Dis. ... Dr. ehkon. Sciences, Ekaterinburg (2013) 